\title{
NORMAL SOLVABILITY OF GENERAL LINEAR ELLIPTIC PROBLEMS
}

\author{
A. VOLPERT AND V. VOLPERT
}

Received 13 June 2004

The paper is devoted to general elliptic problems in the Douglis-Nirenberg sense. We obtain a necessary and sufficient condition of normal solvability in the case of unbounded domains. Along with the ellipticity condition, proper ellipticity and Lopatinsky condition that determine normal solvability of elliptic problems in bounded domains, one more condition formulated in terms of limiting problems should be imposed in the case of unbounded domains.

\section{Introduction}

In this work we study normal solvability of general elliptic problems in the DouglisNirenberg sense. If in the case of bounded domains with a sufficiently smooth boundary the normal solvability is completely determined by the conditions of ellipticity, proper ellipticity, and Lopatinsky condition (see $[2,3,19,20]$ ), then in the case of unbounded domains one more condition related to behavior of solutions at infinity should be imposed.

If the coefficients of the operator have limits at infinity, and the domain is cylindrical or conical at infinity, then the additional condition is determined by the invertibility of limiting operators, that is of the operators with the limiting coefficients in the limiting domain. This situation is studied in a number of works for differential $[4,11,21,25,26]$ and pseudodifferential operators $[15,17,16]$.

If the coefficients do not have limits at infinity but the domain is the whole $R^{n}$, the notion of limiting operators was used in $[12,13]$. Previously it was used in the onedimensional case to study differential equations with quasi-periodic coefficients $[6,8,9$, 14] (see also [18]).

In the case of arbitrary domains, we need to introduce the notion of limiting domains and limiting problems. In the case of general elliptic problems and Hölder spaces it is done in [23]. In [22] we study scalar elliptic problems in Sobolev spaces (see below). We obtain conditions of normal solvability in terms of uniqueness of solutions of limiting problems. In this work we generalize these results to the case of systems.

We should note that the choice of function spaces plays important role. We introduce a generalization of Sobolev-Slobodetskii spaces that will be essentially used in the 
subsequent works where we consider nonhomogeneous adjoint problems, obtain a priori estimates for them and prove their normal solvability. These results are used to prove the Fredholm property of general elliptic problems. For the scalar equation the solvability conditions will be also formulated in terms of formally adjoint problems. We will use the approach developed in [10] for scalar elliptic problems in bounded domains.

1.1. Function spaces. Sobolev spaces $W^{s, p}$ proved to be very convenient in the study of elliptic problems in bounded domains. But more flexible spaces are needed for elliptic problems in unbounded domains. We need some generalization of the space $W^{s, p}$. More exactly, we mean such spaces which coincide with $W^{s, p}$ in bounded domains but have a prescribed behavior at infinity in unbounded domains. It turns out that such spaces can be constructed for arbitrary Banach spaces of distributions (not only Sobolev spaces) as follows.

Consider first functions defined on $R^{n}$. As usual we denote by $D$ the space of infinitely differentiable functions with compact support and by $D^{\prime}$ its dual. Let $E \subset D^{\prime}$ be a Banach space, the inclusion is understood both in algebraic and topological sense. Denote by $E_{\text {loc }}$ the collection of all $u \in D^{\prime}$ such that $f u \in E$ for all $f \in D$. Let $\omega(x) \in D, 0 \leq \omega(x) \leq 1$, $\omega(x)=1$ for $|x| \leq 1 / 2, \omega(x)=0$ for $|x| \geq 1$.

Definition 1.1. $E_{q}(1 \leq q \leq \infty)$ is the space of all $u \in E_{\text {loc }}$ such that

$$
\begin{aligned}
&\|u\|_{E_{q}}:=\left(\int_{R^{n}}\|u(\cdot) \omega(\cdot-y)\|_{E}^{q} d y\right)^{1 / q}<\infty, \quad 1 \leq q<\infty, \\
&\|u\|_{E_{\infty}}:=\sup _{y \in R^{n}}\|u(\cdot) \omega(\cdot-y)\|_{E}<\infty .
\end{aligned}
$$

It is proved that $E_{q}$ is a Banach space. If $\Omega$ is a domain in $R^{n}$, then by definition $E_{q}(\Omega)$ is the space of restrictions of $E_{q}$ to $\Omega$ with the usual norm of restrictions. It is easy to see that if $\Omega$ is a bounded domain, then

$$
E_{q}(\Omega)=E(\Omega), \quad 1 \leq q \leq \infty .
$$

In particular, if $E=W^{s, p}$, then we denote $W_{q}^{s, p}=E_{q}(1 \leq q \leq \infty)$. It is proved that

$$
W_{p}^{s, p}=W^{s, p} \quad(s \geq 0,1<p<\infty) .
$$

Hence the spaces $W_{q}^{s, p}$ generalize the Sobolev spaces $(q<\infty)$ and the Stepanov spaces $(q=\infty)($ see $[8,9,14])$.

If $E=L^{p}$, then $L_{q}^{p}=E_{q}$. It can be proved that if $u$ belongs to $L^{p}$ locally and $|u(x)| \leq$ $K|x|^{-\alpha}$ for $|x|$ sufficiently large, where $K$ is a positive constant, and $\alpha q>n$, then $u \in L_{q}^{p}$. Unlike the spaces $L^{p}$ for which there is no embedding $L^{p}\left(R^{n}\right)$ in $L^{p_{1}}\left(R^{n}\right)$ for any $1<p$, $p_{1}<\infty, p \neq p_{1}$, it is easy to prove that

$$
L_{q}^{p}\left(R^{n}\right) \subset L_{q_{1}}^{p_{1}}\left(R^{n}\right) \quad\left(p \geq p_{1}, q \leq q_{1}\right)
$$


1.2. Elliptic problems. Consider the operators

$$
\begin{aligned}
A_{i} u & =\sum_{k=1}^{N} \sum_{|\alpha| \leq \alpha_{i k}} a_{i k}^{\alpha}(x) D^{\alpha} u_{k}, \quad i=1, \ldots, N, x \in \Omega, \\
B_{j} u & =\sum_{k=1}^{N} \sum_{|\beta| \leq \beta_{j k}} b_{j k}^{\beta}(x) D^{\beta} u_{k}, \quad i=1, \ldots, m, x \in \partial \Omega,
\end{aligned}
$$

where $u=\left(u_{1}, \ldots, u_{N}\right), \Omega \subset R^{n}$ is an unbounded domain that satisfy certain conditions given below. According to the definition of elliptic operators in the Douglis-Nirenberg sense [5] we suppose that

$$
\alpha_{i k} \leq s_{i}+t_{k}, \quad i, k=1, \ldots, N, \quad \beta_{j k} \leq \sigma_{j}+t_{k}, \quad j=1, \ldots, m, k=1, \ldots, N
$$

for some integers $s_{i}, t_{k}, \sigma_{j}$ such that $s_{i} \leq 0, \max s_{i}=0, t_{k} \geq 0$.

Denote by $E$ the space of vector-valued functions $u=\left(u_{1}, \ldots, u_{N}\right)$, where $u_{j}$ belongs to the Sobolev space $W^{l+t_{j}, p}(\Omega), j=1, \ldots, N, 1<p<\infty, l$ is an integer, $l \geq \max \left(0, \sigma_{j}+1\right)$, $E=\Pi_{j=1}^{N} W^{l+t_{j}, p}(\Omega)$. The norm in this space is defined as

$$
\|u\|_{E}=\sum_{j=1}^{N}\left\|u_{j}\right\|_{W^{l+t_{j}, p}(\Omega)} .
$$

The operator $A_{i}$ acts from $E$ to $W^{l-s_{i}, p}(\Omega)$, the operator $B_{j}$ acts from $E$ to $W^{l-\sigma_{j}-1 / p, p}(\partial \Omega)$. Denote

$$
\begin{gathered}
L=\left(A_{1}, \ldots, A_{N}, B_{1}, \ldots, B_{m}\right), \\
F=\prod_{i=1}^{N} W^{l-s_{i}, p}(\Omega) \times \prod_{j=1}^{m} W^{l-\sigma_{j}-1 / p, p}(\partial \Omega) .
\end{gathered}
$$

We will consider the operator $L$ as acting from $E_{\infty}$ to $F_{\infty}$.

Throughout the paper we assume that the operator $L$ satisfies the condition of uniform ellipticity.

1.3. Limiting problems. We recall that the operator is normally solvable with a finite dimensional kernel if and only if it is proper, that is the inverse image of a compact set is compact in any closed bounded set. In this work we obtain necessary and sufficient conditions for a general elliptic operator to satisfy this property. Consider as example the following operator

$$
L u=a(x) u^{\prime \prime}+b(x) u^{\prime}+c(x) u
$$

acting from $H^{2}(R)$ to $L^{2}(R)$. If we assume that there exist limits of the coefficients of the operator at infinity, then we can define the operators

$$
L_{ \pm} u=a_{ \pm} u^{\prime \prime}+b_{ \pm} u^{\prime}+c_{ \pm} u,
$$

where the subscripts + and - denote the limiting values at $+\infty$ and $-\infty$, respectively. As it 
is well known, the operator $L$ satisfies the Fredholm property if and only if the equations $L_{ \pm} u=0$ do not have nonzero bounded solutions. We can easily write down this condition explicitly:

$$
-a_{ \pm} \xi^{2}+b_{ \pm} i \xi+c_{ \pm} \neq 0
$$

if we look for solutions of this equations in the form $u=\exp (i \xi)$.

This simple approach is not applicable for general elliptic problems where limits of the coefficients may not exist and the domain can be arbitrary. In the next section we will define limiting problems in the general case. Construction of limiting domains can be briefly described as follows. Let $x_{k} \in \Omega$ be a sequence, which tends to infinity. Consider the shifted domains $\Omega_{k}$ corresponding to the shifted characteristic functions $\chi\left(x+x_{k}\right)$, where $\chi(x)$ is the characteristic function of the domain $\Omega$. Consider a ball $B_{r} \subset R^{n}$ with the center at the origin and with the radius $r$. Suppose that for all $k$ there are points of the boundaries $\partial \Omega_{k}$ inside $B_{r}$. If the boundaries are sufficiently smooth, we can expect that from the sequence $\Omega_{k} \cap B_{r}$ we can choose a subsequence that converges to some limiting domain $\Omega_{*}$. After that we take a larger ball and choose a convergent subsequence of the previous subsequence. The usual diagonal process allows us to extend the limiting domain to the whole space.

To define limiting operators we consider shifted coefficients $a^{\alpha}\left(x+x_{k}\right), b_{j}^{\alpha}\left(x+x_{k}\right)$ and choose subsequences that converge to some limiting functions $\hat{a}^{\alpha}(x), \hat{b}_{j}^{\alpha}(x)$ uniformly in every bounded set. The limiting operator is the operator with the limiting coefficients. Limiting operators and limiting domains constitute limiting problems. It is clear that the same problem can have a family of limiting problems depending on the choice of the sequence $x_{k}$ and on the choice of both converging subsequences of domains and coefficients.

We note that in the case where $\Omega=R^{n}$ the limiting domain is also $R^{n}$. In this case the limiting operators were introduced and used in $[12,13,17,18]$.

1.4. Normal solvability. The following condition determines normal solvability of elliptic problems.

Condition NS. Any limiting problem

$$
\hat{L} u=0, \quad x \in \Omega_{*}, u \in E_{\infty}\left(\Omega_{*}\right)
$$

has only zero solution.

It is a necessary and sufficient condition for general elliptic operators considered in Hölder spaces to be normally solvable with a finite dimensional kernel [23]. For scalar elliptic problems in Sobolev spaces it was proved in [22]. In this work we generalize these results for elliptic systems. More precisely, we prove that the elliptic operator $L$ is normally solvable and has a finite-dimensional kernel in the space $W_{\infty}^{l, p}(1<p<\infty)$ if and only if Condition NS is satisfied. Using this result it can be proved that the elliptic operator $L$ is Fredholm (if the limiting operators are invertible) in the space $W_{q}^{l, p}$ for $1<p<\infty$ and some $q$. This result will be published elsewhere. 
It is easy to see how this condition is related to the condition formulated in terms of the Fourier transform. In fact, for operator (1.9) the nonzero solution of the limiting problem $L_{ \pm} u=0$ has the form $u_{0}(x)=e^{i \xi x}$, where $\xi$ is the value for which the essential spectrum passes through 0 . The function $u_{0}(x)$ belongs obviously to the Hölder spaces and also to the space $W_{\infty}^{2, p}(R)$. However it does not belong to the usual Sobolev space $W^{2, p}(R)$. So Condition NS cannot be obtained in terms of usual Sobolev spaces (see also [22] for counter-examples in $R^{n}$ ). This is one of the reasons why it is more convenient to work with $W_{q}^{s, p}$ spaces.

\section{A priori estimates in the spaces $W_{\infty}^{s, p}$}

In this section, we define the spaces $W_{\infty}^{s, p}$ and obtain a priori estimates of solutions, which are similar to those in usual Sobolev spaces.

Denote by $W_{\infty}^{k, p}(\Omega)$ the space of functions defined as the closure of smooth functions in the norm

$$
\|u\|_{W_{\infty}^{k, p}(\Omega)}=\sup _{y \in \Omega}\|u\|_{W^{k, p}\left(\Omega \cap Q_{y}\right)} .
$$

Here $\Omega$ is a domain in $R^{n}, Q_{y}$ is a unit ball with the center at $y,\|\cdot\|_{W^{k, p}}$ is the Sobolev norm. We note that in bounded domains $\Omega$ the norms of the spaces $W^{k, p}(\Omega)$ and $W_{\infty}^{k, p}(\Omega)$ are equivalent. In the one-dimensional case with $k=0$ similar spaces were used in $[8,9$, 14]. This definition is equivalent to Definition 1.1 .

We suppose that the boundary $\partial \Omega$ belongs to the Hölder space $C^{k+\theta}, 0<\theta<1$, and that the Hölder norms of the corresponding functions in local coordinates are bounded independently of the point of the boundary. Then we can define the space $W_{\infty}^{k-1 / p, p}(\partial \Omega)$ of traces on the boundary $\partial \Omega$ of the domain $\Omega$,

$$
\|\phi\|_{W_{\infty}^{k-1 / p, p}(\partial \Omega)}=\inf \|v\|_{W_{\infty}^{k, p}(\Omega)},
$$

where the infimum is taken with respect to all functions $v \in W_{\infty}^{k, p}(\Omega)$ equal $\phi$ at the boundary, and $k>1 / p$.

The space $W_{\infty}^{k, p}(\Omega)$ with $k=0$ will be denoted by $L_{\infty}^{p}(\Omega)$. We will use also the notations

$$
\begin{gathered}
E_{\infty}=\Pi_{j=1}^{N} W_{\infty}^{l+t_{j}, p}(\Omega), \\
F_{\infty}=\prod_{i=1}^{N} W_{\infty}^{l-s_{i}, p}(\Omega) \times \prod_{j=1}^{m} W_{\infty}^{l-\sigma_{j}-1 / p, p}(\partial \Omega) .
\end{gathered}
$$

We consider the operator $L$ defined by $(1.8)$ and denote $l_{1}=\max \left(0, \sigma_{j}+1\right)$. We suppose that the integer $l$ in the definition of the spaces is such that $l \geq l_{1}$, and the boundary $\partial \Omega$ belongs to the class $C^{r+\theta}$ with $r$ specified in Condition $\mathrm{D}$ below.

Theorem 2.1. Let $u \in \Pi_{j=1}^{N} W_{\infty}^{l_{1}+t_{j}, p}(\Omega)$. Then for any $l \geq l_{1}$ we have $u \in E_{\infty}$ and

$$
\|u\|_{E_{\infty}} \leq c\left(\|L u\|_{F_{\infty}}+\|u\|_{L_{\infty}^{p}(\Omega)}\right),
$$

where the constant $c$ does not depend on $u$. 
738 Normal solvability of general linear elliptic problems

Proof. Let $\omega(x)$ be an infinitely differentiable nonnegative function such that

$$
\omega(x)=1, \quad|x| \leq \frac{1}{2}, \quad \omega(x)=0, \quad|x| \geq 1 .
$$

Denote $\omega_{y}(x)=\omega(x-y)$. Suppose $u(x)$ is a function satisfying the conditions of the theorem. Then $\omega_{y} u \in \Pi_{j=1}^{N} W_{\infty}^{l_{1}+t_{j}, p}(\Omega)$. Since the support of this function is bounded, we can use now a priori estimates of solutions [1]:

$$
\left\|\omega_{y} u\right\|_{E} \leq c\left(\left\|L\left(\omega_{y} u\right)\right\|_{F}+\left\|\omega_{y} u\right\|_{L^{p}(\Omega)}\right), \quad \forall y \in R^{n},
$$

where the constant $c$ does not depend on $y$. We now estimate the right-hand side of the last inequality. We have

$$
A_{i}\left(\omega_{y} u\right)=\omega_{y} A_{i} u+T_{i}
$$

where

$$
T_{i}=\sum_{k=1}^{N} \sum_{|\alpha| \leq \alpha_{i k}} a_{i k}^{\alpha} \sum_{\beta+\gamma \leq \alpha,|\beta|>0} c_{\beta \gamma} D^{\beta} \omega_{y} D^{\gamma} u_{k},
$$

and $c_{\beta \gamma}$ are some constants. If $|\tau| \leq l-s_{i}$, then

$$
\left\|D^{\tau}\left(\omega_{y} A_{i} u\right)\right\|_{L^{p}(\Omega)} \leq M\left\|A_{i} u\right\|_{W_{\infty}^{l-s_{i}, p}(\Omega)} .
$$

For any $\epsilon>0$ we have the estimate

$$
\begin{aligned}
\left\|T_{i}\right\|_{W^{l-s_{i}, p}(\Omega)} & \leq \epsilon \sum_{k=1}^{N}\left\|u_{k}\right\|_{W^{l+t_{k}, p}\left(\Omega \cap Q_{y}\right)}+C_{\epsilon} \sum_{k=1}^{N}\left\|u_{k}\right\|_{L^{p}\left(\Omega \cap Q_{y}\right)} \\
& \leq \epsilon\|u\|_{E_{\infty}}+C_{\epsilon}\|u\|_{L_{\infty}^{p}(\Omega)},
\end{aligned}
$$

where $Q_{y}$ is a unit ball with the center at $y$.

Thus

$$
\left\|A_{i}\left(\omega_{y} u\right)\right\|_{W^{l-s_{i}, p}(\Omega)} \leq M\left\|A_{i} u\right\|_{W_{\infty}^{l-s_{i}, p}(\Omega)}+\epsilon\|u\|_{E_{\infty}}+C_{\epsilon}\|u\|_{L_{\infty}^{p}(\Omega)} .
$$

Consider next the boundary operators in the right-hand side of (2.6). We have

$$
B_{j}\left(\omega_{y} u\right)=\omega_{y} \Phi_{j}+S_{j}
$$

where $\Phi_{j}=B_{j} u$,

$$
S_{j}=\sum_{k=1}^{N} \sum_{|\beta| \leq \beta_{j k}} b_{j k}^{\beta} \sum_{\alpha+\gamma \leq \beta,|\alpha|>0} \lambda_{\alpha \gamma} D^{\alpha} \omega_{y} D^{\gamma} u_{k}
$$

and $\lambda_{\alpha \gamma}$ are some constants. 
There exists a function $v \in W_{\infty}^{l-\sigma_{j}, p}(\Omega)$ such that $v=\Phi_{i}$ on $\partial \Omega$ and

$$
\|v\|_{W_{\infty}^{l-\sigma_{j}, p}(\Omega)} \leq 2\left\|\Phi_{j}\right\|_{W_{\infty}^{l-\sigma_{j}-1 / p, p}(\partial \Omega)} .
$$

Since $v \in W_{\infty}^{l-\sigma_{j}, p}(\Omega)$, then $\omega_{y} v \in W^{l-\sigma_{j}, p}(\Omega)$ and

$$
\left\|\omega_{y} v\right\|_{W^{l-\sigma_{j}, p}(\Omega)} \leq M\|v\|_{W_{\infty}^{l-\sigma_{j}, p}(\Omega)}
$$

with a constant $M$ independent of $v$. Since $\omega_{y} v=\omega_{y} \Phi_{j}$ on $\partial \Omega$, then

$$
\left\|\omega_{j} \Phi_{j}\right\|_{W^{l-\sigma_{j}-1 / p, p}(\partial \Omega)} \leq M_{1}\left\|\Phi_{j}\right\|_{W_{\infty}^{l-\sigma_{j}-1 / p, p}(\partial \Omega)} .
$$

Further,

$$
\begin{aligned}
\left\|S_{j}\right\|_{W^{l-\sigma_{j}-1 / p, p}(\partial \Omega)} & \leq\left\|S_{j}\right\|_{W^{l-\sigma_{j}, p}(\Omega)} \leq \epsilon \sum_{k=1}^{N}\left\|u_{k}\right\|_{W^{l+t_{k}, p}\left(\Omega \cap Q_{y}\right)}+C_{\epsilon} \sum_{k=1}^{N}\left\|u_{k}\right\|_{L^{p}\left(\Omega \cap Q_{y}\right)} \\
& \leq \epsilon\|u\|_{E_{\infty}}+C_{\epsilon}\|u\|_{L_{\infty}^{p}(\Omega)} .
\end{aligned}
$$

Thus

$$
\left\|B_{j}\left(\omega_{y} u\right)\right\|_{W^{l-\sigma_{j}-1 / p, p}(\partial \Omega)} \leq M\left\|\Phi_{j}\right\|_{W_{\infty}^{l-\sigma_{j}-1 / p, p}(\partial \Omega)}+\epsilon\|u\|_{E_{\infty}}+C_{\epsilon}\|u\|_{L_{\infty}^{p}(\Omega)} .
$$

From (2.6), (2.11), and (2.18) we obtain the estimate

$$
\left\|\omega_{y} u\right\|_{E} \leq c\left(M_{2}\|L u\|_{F_{\infty}}+\kappa \epsilon\|u\|_{E_{\infty}}+C_{\epsilon}\|u\|_{L_{\infty}^{p}(\Omega)}\right)
$$

with some constants $M_{2}$ and $\kappa$. Taking $\epsilon>0$ sufficiently small, we obtain (2.4). The theorem is proved.

\section{Limiting problems}

In this section, we define limiting domains and limiting operators. They determine limiting problems. We will restrict ourselves to the definitions and to the result, which we give without proofs, that will be used below. More detailed presentation including the proofs can be found in [22].

3.1. Limiting domains. In this section, we define limiting domains for unbounded domains in $R^{n}$, show their existence and study some of their properties. We consider an unbounded domain $\Omega \subset R^{n}$, which satisfies the following condition.

Condition $D$. For each $x_{0} \in \partial \Omega$ there exists a neighborhood $U\left(x_{0}\right)$ such that:

(1) $U\left(x_{0}\right)$ contains a sphere with the radius $\delta$ and the center $x_{0}$, where $\delta$ is independent of $x_{0}$, 
(2) there exists a homeomorphism $\psi\left(x ; x_{0}\right)$ of the neighborhood $U\left(x_{0}\right)$ on the unit sphere $B=\{y:|y|<1\}$ in $R^{n}$ such that the images of $\Omega \cap U\left(x_{0}\right)$ and $\partial \Omega \cap U\left(x_{0}\right)$ coincide with $B_{+}=\left\{y: y_{n}>0,|y|<1\right\}$ and $B_{0}=\left\{y: y_{n}=0,|y|<1\right\}$, respectively,

(3) the function $\psi\left(x ; x_{0}\right)$ and its inverse belong to the Hölder space $C^{r+\theta}, 0<\theta<1$. Their $\|\cdot\|_{r+\theta}$-norms are bounded uniformly in $x_{0}$.

For definiteness we suppose that $\delta<1$. We assume also that

$$
r \geq \max \left(l+t_{i}, l-s_{i}, l-\sigma_{j}+1\right), \quad i=1, \ldots, N, j=1, \ldots, m .
$$

The first expression in the maximum is used for a priori estimates of solutions, the second and the third will allow us to extend the coefficients of the operator (see Section 3.3).

To define convergence of domains we use the following Hausdorff metric space. Let $M$ and $N$ denote two nonempty closed sets in $R^{n}$. Denote

$$
\varsigma(M, N)=\sup _{a \in M} \rho(a, N), \quad \varsigma(N, M)=\sup _{b \in N} \rho(b, M),
$$

where $\rho(a, N)$ denotes the distance from a point $a$ to a set $N$, and let

$$
\varrho(M, N)=\max (\varsigma(M, N), \varsigma(N, M))
$$

We denote by $\Xi$ a metric space of bounded closed nonempty sets in $R^{n}$ with the distance given by (3.3). We say that a sequence of domains $\Omega_{m}$ converges to a domain $\Omega$ in $\Xi_{\text {loc }}$ if

$$
\varrho\left(\bar{\Omega}_{m} \cap \bar{B}_{R}, \bar{\Omega} \cap \bar{B}_{R}\right) \longrightarrow 0, \quad m \longrightarrow \infty
$$

for any $R>0$ and $B_{R}=\{x:|x|<R\}$. Here the bar denotes the closure of domains.

Definition 3.1. Let $\Omega \subset R^{n}$ be an unbounded domain, $x_{m} \in \Omega,\left|x_{m}\right| \rightarrow \infty$ as $m \rightarrow \infty ; \chi(x)$ be the characteristic function of $\Omega$, and $\Omega_{m}$ be a shifted domain defined by the characteristic function $\chi_{m}(x)=\chi\left(x+x_{m}\right)$. We say that $\Omega_{*}$ is a limiting domain of the domain $\Omega$ if $\Omega_{m} \rightarrow \Omega_{*}$ in $\Xi_{\text {loc }}$ as $m \rightarrow \infty$.

We denote by $\Lambda(\Omega)$ the set of all limiting domains of the domain $\Omega$ (for all sequences $\left.x_{m}\right)$. We will show below that if Condition $\mathrm{D}$ is satisfied, then the limiting domains exist and also satisfy this condition.

Theorem 3.2. If a domain $\Omega$ satisfies Condition $D$, then there exists a function $f(x)$ defined in $R^{n}$ such that:

(1) $f(x) \in C^{k+\theta}\left(R^{n}\right), k \geq r$,

(2) $f(x)>0$ if and only if $x \in \Omega$,

(3) $|\nabla f(x)| \geq 1$ for $x \in \partial \Omega$,

(4) $\min (d(x), 1) \leq|f(x)|$, where $d(x)$ is the distance from $x$ to $\partial \Omega$.

Let $\Omega$ be an unbounded domain satisfying Condition $\mathrm{D}$ and $f(x)$ be a function satisfying conditions of Theorem 3.2. Consider a sequence $x_{m} \in \Omega,\left|x_{m}\right| \rightarrow \infty$. Denote

$$
f_{m}(x)=f\left(x+x_{m}\right) \text {. }
$$


Theorem 3.3. Let $f_{m}(x) \rightarrow f_{*}(x)$ in $C_{\mathrm{loc}}^{k}\left(R^{n}\right)$, where $k$ is not greater than that in Theorem 3.2. Denote

$$
\Omega_{*}=\left\{x: x \in R^{n}, f_{*}(x)>0\right\}
$$

Then

(1) $f_{*}(x) \in C^{k+\theta}\left(R^{n}\right)$,

(2) $\Omega_{*}$ is an nonempty open set.

If $\Omega_{*} \neq R^{n}$, then

(3) $\left|\nabla f_{*}(x)\right|_{\partial \Omega_{*}} \geq 1$,

(4) $\min \left(d_{*}(x), 1\right) \leq\left|f_{*}(x)\right|$, where $d_{*}(x)$ is the distance from $x$ to $\partial \Omega_{*}$.

Theorem 3.4. If $f_{m}(x) \rightarrow f_{*}(x)$ in $C_{\mathrm{loc}}^{k}$ as $m \rightarrow \infty$, then $\partial \Omega_{m} \rightarrow \partial \Omega_{*}$ in $\Xi_{\mathrm{loc}}$. Moreover, the limiting domain $\Omega_{*}$ either satisfies Condition $D$ or $\Omega_{*}=R^{n}$.

Theorem 3.5. Let $\Omega$ be an unbounded domain satisfying Condition $D, x_{m} \in \Omega,\left|x_{m}\right| \rightarrow \infty$, and $f(x)$ be the function constructed in Theorem 3.2.

Then there exists a subsequence $x_{m_{i}}$ and a function $f_{*}(x)$ such that

$$
f_{m_{i}}(x) \equiv f\left(x+x_{m_{i}}\right) \longrightarrow f_{*}(x)
$$

in $C_{\mathrm{loc}}^{k}\left(R^{n}\right)$, and the domain $\Omega_{*}=\left\{x: f_{*}(x)>0\right\}$ either satisfies Condition D or $\Omega_{*}=R^{n}$. Moreover, $\bar{\Omega}_{m_{i}} \rightarrow \bar{\Omega}_{*}$ in $\Xi_{\text {loc }}$, where $\Omega_{m_{i}}=\left\{x: f_{m_{i}}(x)>0\right\}$.

3.2. Convergence. In the previous section we have introduced limiting domains. Here we define the corresponding limiting problems.

Let $\Omega$ be a domain satisfying Condition $\mathrm{D}$ and $\chi(x)$ be its characteristic function. Consider a sequence $x_{m} \in \Omega,\left|x_{m}\right| \rightarrow \infty$ and the shifted domains $\Omega_{m}$ defined by the shifted characteristic functions $\chi_{m}(x)=\chi\left(x+x_{m}\right)$. We suppose that the sequence of domains $\Omega_{m}$ converge in $\Xi_{\text {loc }}$ to some limiting domain $\Omega_{*}$. In this section we suppose that $0 \leq k \leq r$.

Definition 3.6. Let $u_{m} \in W_{\infty}^{k, p}\left(\Omega_{m}\right), m=1,2, \ldots$ We say that $u_{m}$ converges to a limiting function $u_{*} \in W_{\infty}^{k, p}\left(\Omega_{*}\right)$ in $W_{\text {loc }}^{k, p}\left(\Omega_{m} \rightarrow \Omega_{*}\right)$ if there exists an extension $v_{m}(x) \in$ $W_{\infty}^{k, p}\left(R^{n}\right)$ of $u_{m}(x), m=1,2, \ldots$ and an extension $v_{*}(x) \in W_{\infty}^{k, p}\left(R^{n}\right)$ of $u_{*}(x)$ such that $v_{m} \rightarrow v_{*}$ in $W_{\mathrm{loc}}^{k, p}\left(R^{n}\right)$.

Definition 3.7. Let $u_{m} \in W_{\infty}^{k-1 / p, p}\left(\partial \Omega_{m}\right), k>1 / p, m=1,2, \ldots$. We say that $u_{m}$ converges to a limiting function $u_{*} \in W_{\infty}^{k-1 / p, p}\left(\partial \Omega_{*}\right)$ in $W_{\text {loc }}^{k-1 / p, p}\left(\partial \Omega_{m} \rightarrow \partial \Omega_{*}\right)$ if there exists an extension $v_{m}(x) \in W_{\infty}^{k, p}\left(R^{n}\right)$ of $u_{m}(x), m=1,2, \ldots$ and an extension $v_{*}(x) \in W_{\infty}^{k, p}\left(R^{n}\right)$ of $u_{*}(x)$ such that $v_{m} \rightarrow v_{*}$ in $W_{\mathrm{loc}}^{k, p}\left(R^{n}\right)$.

Definition 3.8. Let $u_{m}(x) \in C^{k}\left(\Omega_{m}\right), m=1,2, \ldots$ We say that $u_{m}(x)$ converges to a limiting function $u_{*}(x) \in C^{k}\left(\Omega_{*}\right)$ in $C_{\text {loc }}^{k}\left(\Omega_{m} \rightarrow \Omega_{*}\right)$ if there exists an extension $v_{m}(x) \in$ $C^{k}\left(R^{n}\right)$ of $u_{m}(x), m=1,2, \ldots$ and an extension $v_{*}(x) \in C^{k}\left(R^{n}\right)$ of $u_{*}(x)$ such that

$$
v_{m} \longrightarrow v_{*} \quad \text { in } C_{\mathrm{loc}}^{k}\left(R^{n}\right)
$$


742 Normal solvability of general linear elliptic problems

Definition 3.9. Let $u_{m}(x) \in C^{k}\left(\partial \Omega_{m}\right), m=1,2, \ldots$ We say that $u_{m}(x)$ converges to a limiting function $u_{*}(x) \in C^{k}\left(\partial \Omega_{*}\right)$ in $C_{\mathrm{loc}}^{k}\left(\partial \Omega_{m} \rightarrow \partial \Omega_{*}\right)$ if there exists an extension $v_{m}(x) \in$ $C^{k}\left(R^{n}\right)$ of $u_{m}(x), m=1,2, \ldots$ and an extension $v_{*}(x) \in C^{k}\left(R^{n}\right)$ of $u_{*}(x)$ such that

$$
v_{m} \longrightarrow v_{*} \quad \text { in } C_{\text {loc }}^{k}\left(R^{n}\right) .
$$

Theorem 3.10. The limiting function $u_{*}(x)$ in Definitions 3.6-3.9 does not depend on the choice of extensions $v_{m}(x)$ and $v_{*}(x)$.

Theorem 3.11. Suppose that $0<k \leq r-1$. Let

$$
u_{m} \in W_{\infty}^{k+1, p}\left(\Omega_{m}\right), \quad\left\|u_{m}\right\|_{W_{\infty}^{k+1, p}\left(\Omega_{m}\right)} \leq M,
$$

where the constant $M$ does not depend on $m$. Then there exists a function $u_{*} \in W_{\infty}^{k+1, p}\left(\Omega_{*}\right)$ and a subsequence $u_{m_{i}}$ such that $u_{m_{i}} \rightarrow u_{*}$ in $W_{\mathrm{loc}}^{k, p}\left(\Omega_{m} \rightarrow \Omega_{*}\right)$.

Theorem 3.12. Suppose that $0<k \leq r-1$. Let $u_{m} \in W_{\infty}^{k+1-1 / p, p}\left(\partial \Omega_{m}\right)$,

$$
\left\|u_{m}\right\|_{W_{\infty}^{k+1-1 / p, p}\left(\partial \Omega_{m}\right)} \leq M,
$$

where the constant $M$ does not depend on $m$. Then there exists a function

$$
u_{*} \in W_{\infty}^{k+1-1 / p, p}\left(\partial \Omega_{*}\right)
$$

and a subsequence $u_{m_{i}}$ such that

$$
u_{m_{i}} \longrightarrow u_{*} \quad \text { in } W_{\mathrm{loc}}^{k+1-\epsilon-1 / p, p}\left(\partial \Omega_{m} \longrightarrow \partial \Omega_{*}\right),
$$

where $0<\epsilon<k+1-1 / p$.

Theorem 3.13. Let $u_{m} \in C^{k+\theta}\left(\Omega_{m}\right),\left\|u_{m}\right\|_{C^{k+\theta}} \leq M$, where the constant $M$ is independent of $m$. Then there exists a function $u_{*} \in C^{k+\theta}\left(\Omega_{*}\right)$ and a subsequence $u_{m_{k}}$ such that $u_{m_{k}} \rightarrow u_{*}$ in $C_{\mathrm{loc}}^{k}\left(\Omega_{m_{k}} \rightarrow \Omega_{*}\right)$.

Let $u_{m} \in C^{k+\theta}\left(\partial \Omega_{m}\right),\left\|u_{m}\right\|_{C^{k+\theta}} \leq M$. Then there exists a function $u_{*} \in C^{k+\theta}\left(\partial \Omega_{*}\right)$ and a subsequence $u_{m_{k}}$ such that $u_{m_{k}} \rightarrow u_{*}$ in $C_{\mathrm{loc}}^{k}\left(\partial \Omega_{m_{k}} \rightarrow \partial \Omega_{*}\right)$.

3.3. Limiting operators. Suppose that we are given a sequence $\left\{x_{\nu}\right\}, v=1,2, \ldots, x_{\nu} \in$ $\Omega,\left|x_{\nu}\right| \rightarrow \infty$. Consider the shifted domains $\Omega_{\nu}$ with the characteristic functions $\chi_{\nu}(x)=$ $\chi\left(x+x_{v}\right)$ where $\chi(x)$ is the characteristic function of $\Omega$, and the shifted coefficients of the operators $A_{i}$ and $B_{j}$ :

$$
a_{i k, v}^{\alpha}(x)=a_{i k}^{\alpha}\left(x+x_{\nu}\right), \quad b_{j k, v}^{\beta}(x)=b_{j k}^{\beta}\left(x+x_{\nu}\right) .
$$

We suppose that

$$
a_{i k}^{\alpha}(x) \in C^{l-s_{i}+\theta}(\bar{\Omega}), \quad b_{j k}^{\beta}(x) \in C^{l-\sigma_{j}+\theta}(\partial \Omega),
$$


where $0<\theta<1$, and that these coefficients can be extended to $R^{n}$ :

$$
a_{i k}^{\alpha}(x) \in C^{l-s_{i}+\theta}\left(R^{n}\right), \quad b_{j k}^{\beta}(x) \in C^{l-\sigma_{j}+\theta}\left(R^{n}\right) .
$$

Therefore

$$
\left\|a_{i k, v}^{\alpha}(x)\right\|_{C^{l-s_{i}+\theta}\left(R^{n}\right)} \leq M, \quad\left\|b_{j k, v}^{\beta}(x)\right\|_{C^{l-\sigma_{j}+\theta}\left(R^{n}\right)} \leq M
$$

with some constant $M$ independent of $v$. It follows from Theorem 3.5 that there exists a subsequence of the sequence $\Omega_{\gamma}$, for which we keep the same notation, such that it converges to a limiting domain $\Omega_{*}$. From (3.17) it follows that this subsequence can be chosen such that

$$
a_{i k, v}^{\alpha} \longrightarrow \hat{a}_{i k}^{\alpha} \quad \text { in } C^{l-s_{i}}\left(R^{n}\right) \text { locally, } \quad b_{j k, v}^{\beta} \longrightarrow \hat{b}_{j k}^{\beta} \quad \text { in } C^{l-\sigma_{j}}\left(R^{n}\right) \text { locally, }
$$

where $\hat{a}_{i k}^{\alpha}$ and $\hat{b}_{j k}^{\beta}$ are limiting coefficients,

$$
\hat{a}_{i k}^{\alpha} \in C^{l-s_{i}+\theta}\left(R^{n}\right), \quad \hat{b}_{j k}^{\beta} \in C^{l-\sigma_{j}+\theta}\left(R^{n}\right) .
$$

We have constructed limiting operators:

$$
\begin{gathered}
\hat{A}_{i} u=\sum_{k=1}^{N} \sum_{|\alpha| \leq \alpha_{i k}} \hat{a}_{i k}^{\alpha}(x) D^{\alpha} u_{k}, \quad i=1, \ldots, N, x \in \Omega_{*}, \\
\hat{B}_{j} u=\sum_{k=1}^{N} \sum_{|\beta| \leq \beta_{j k}} \hat{b}_{j k}^{\beta}(x) D^{\beta} u_{k}, \quad i=1, \ldots, m, x \in \partial \Omega_{*}, \\
\hat{L}=\left(\hat{A}_{1}, \ldots, \hat{A}_{N}, \hat{B}_{1}, \ldots, \hat{B}_{m}\right) .
\end{gathered}
$$

We consider them as acting from $E_{\infty}\left(\Omega_{*}\right)$ to $F_{\infty}\left(\Omega_{*}\right)$.

\section{A priori estimates with condition ns}

In Section 5, we will prove that Condition NS (Section 1.4) is necessary and sufficient in order for the operator $L$ to be normal solvable with a finite dimensional kernel. In this section we will use it to obtain a priori estimates of solutions stronger than those given by Theorem 2.1. Estimates of this type are first obtained in $[12,13]$ for elliptic operators in the whole $R^{n}$.

Theorem 4.1. Let Condition NS be satisfied. Then there exist numbers $M_{0}$ and $R_{0}$ such that the following estimate holds:

$$
\|u\|_{E_{\infty}} \leq M_{0}\left(\|L u\|_{F_{\infty}}+\|u\|_{L^{p}\left(\Omega_{R_{0}}\right)}\right), \quad \forall u \in E_{\infty} .
$$

Here $\Omega_{R_{0}}=\Omega \cap\left\{|x| \leq R_{0}\right\}$. 
744 Normal solvability of general linear elliptic problems

Proof. Suppose that the assertion of the theorem is not right. Let $M_{k} \rightarrow \infty$ and $R_{k} \rightarrow \infty$ be given sequences. Then there exists $u_{k} \in E_{\infty}$ such that

$$
\left\|u_{k}\right\|_{E_{\infty}}>M_{k}\left(\left\|L u_{k}\right\|_{F_{\infty}}+\left\|u_{k}\right\|_{L^{p}\left(\Omega_{R_{k}}\right)}\right)
$$

We can suppose that

$$
\left\|u_{k}\right\|_{E_{\infty}}=1
$$

Then

$$
\left\|L u_{k}\right\|_{F_{\infty}}+\left\|u_{k}\right\|_{L^{p}\left(\Omega_{R_{k}}\right)}<\frac{1}{M_{k}} \longrightarrow 0 \quad \text { as } k \longrightarrow \infty .
$$

From Theorem 2.1 we obtain

$$
\left\|L u_{k}\right\|_{F_{\infty}}+\left\|u_{k}\right\|_{L_{\infty}^{p}(\Omega)} \geq \frac{1}{c} .
$$

It follows from (4.4) that $\left\|L u_{k}\right\|_{F_{\infty}} \rightarrow 0$. Hence

$$
\left\|u_{k}\right\|_{L_{\infty}^{p}(\Omega)}>\frac{1}{2 c} \quad \text { for } k \geq k_{0}
$$

with some $k_{0}$. Since

$$
\left\|u_{k}\right\|_{L_{\infty}^{p}(\Omega)}=\sup _{y \in \Omega}\left\|u_{k}\right\|_{L^{p}\left(Q_{y} \cap \Omega\right)}
$$

then it follows from (4.6) that there exists $y_{k} \in \Omega$ such that

$$
\left\|u_{k}\right\|_{L^{p}\left(Q_{y_{k}} \cap \Omega\right)}>\frac{1}{2 c} .
$$

From (4.4)

$$
\left\|u_{k}\right\|_{L^{p}\left(\Omega_{R_{k}}\right)} \longrightarrow 0
$$

This convergence and (4.8) imply that $\left|y_{k}\right| \rightarrow \infty$.

Denote

$$
L u_{k}=f_{k}
$$

From (4.4) we get

$$
\left\|f_{k}\right\|_{F_{\infty}} \longrightarrow 0 \text { as } k \longrightarrow \infty \text {. }
$$

Denote next $x=y+y_{k}$,

$$
w_{k}(y)=u_{k}\left(y+y_{k}\right) .
$$


We rewrite (4.10) in the detailed form

$$
\begin{aligned}
& \sum_{h=1}^{N} \sum_{|\alpha| \leq \alpha_{i h}} a_{i h}^{\alpha}(x) D^{\alpha} u_{h k}=f_{i k}, \quad i=1, \ldots, N, x \in \Omega, \\
& \sum_{h=1}^{N} \sum_{|\beta| \leq \beta_{j h}} b_{j h}^{\beta}(x) D^{\beta} u_{h k}=f_{j}^{b}, \quad i=1, \ldots, m, x \in \partial \Omega,
\end{aligned}
$$

where

$$
f_{k}=\left(f_{1_{k}}, \ldots, f_{N_{k}}, f_{1 k}^{b}, \ldots, f_{m k}^{b}\right), \quad u_{k}=\left(u_{1 k}, \ldots, u_{N k}\right)
$$

Denoting

$$
a_{i h}^{\alpha}(y)=a_{i h}^{\alpha}\left(y+y_{k}\right), \quad b_{j h}^{\beta}(y)=b_{j h}^{\beta}\left(y+y_{k}\right),
$$

we obtain from (4.13)

$$
\begin{gathered}
\sum_{h=1}^{N} \sum_{|\alpha| \leq \alpha_{i h}} a_{i h k}^{\alpha}(y) D^{\alpha} w_{h k}(y)=f_{i k}\left(y+y_{k}\right), \quad i=1, \ldots, N, x \in \Omega_{k}, \\
\sum_{h=1}^{N} \sum_{|\beta| \leq \beta_{j h}} b_{j h_{k}}^{\beta}(y) D^{\beta} w_{h k}(y)=f_{j}^{b}\left(y+y_{k}\right), \quad i=1, \ldots, m, x \in \partial \Omega_{k},
\end{gathered}
$$

$\Omega_{k}$ is the shifted domain. From (4.3) we have

$$
\left\|w_{k}\right\|_{E_{\infty}\left(\Omega_{k}\right)}=1
$$

We have $w_{k}=\left(w_{1 k}, \ldots, w_{N k}\right)$, and (4.18) can be written in the form

$$
\sum_{i=1}^{N}\left\|w_{i k}\right\|_{W_{\infty}^{l+t_{i}, p}\left(\Omega_{k}\right)}=1 .
$$

We suppose that $w_{i k}$ are extended to $R^{n}$ such that their $W_{\infty}^{l+t_{i}, p}\left(R^{n}\right)$-norms are uniformly bounded. Passing to a subsequence and retaining the same notation, we can suppose that

$$
\begin{gathered}
w_{i k} \longrightarrow w_{i 0} \quad \text { in } W^{l+t_{i}-\epsilon, p}\left(R^{n}\right) \text { locally, } \quad(\epsilon>0), \\
w_{i k} \longrightarrow w_{i 0} \quad \text { in } W^{l+t_{i}, p}\left(R^{n}\right) \text { locally weakly }
\end{gathered}
$$

for some $w_{i 0}$ as $k \rightarrow \infty$, and

$$
w_{i 0} \in W_{\infty}^{l+t_{i}, p}\left(R^{n}\right), \quad i=1, \ldots, N .
$$


Denote $w_{0}=\left(w_{10}, \ldots, w_{N_{0}}\right)$. We prove that

$$
\hat{L} w_{0}=0
$$

for a limiting operator $\hat{L}$. To do this we pass to the limit in (4.16), (4.17) by a subsequence of $k$. We choose this subsequence such that $\Omega_{k}$ converges to a limiting domain, $\Omega_{k} \rightarrow \Omega_{*}$, and keep for it the same notation.

We begin with (4.16). For any $x_{0} \in \Omega_{*}$ we take a neighborhood $U$ in such a way that $U \subset \Omega_{k}$ for $k$ sufficiently large. For any $\phi \in D$ with the support in $U$ we get from (4.16):

$$
\int_{U} \sum_{h=1}^{N} \sum_{|\alpha| \leq \alpha_{i h}} a_{i h}^{\alpha}(y) D^{\alpha} w_{h k}(y) \phi(y) d y=\int_{U} f_{i_{k}}\left(y+y_{k}\right) \phi(y) d y .
$$

We can suppose, passing to a subsequence, that

$$
a_{i h, k}^{\alpha}(y) \longrightarrow \hat{a}_{i h}^{\alpha}(y) \quad \text { in } C^{l-s_{i}}\left(R^{n}\right) \text { locally }
$$

(see (3.17)), where $\hat{a}_{i h}^{\alpha}(y)$ are the coefficients of the limiting operator. It follows from (4.21) that $D^{\alpha} w_{h k}\left(|\alpha| \leq \alpha_{i h}\right)$ converges locally weakly in $W^{l-s_{i}, p}$ to $D^{\alpha} w_{h 0}$ as $k \rightarrow \infty$. Hence we can pass to the limit in (4.24).

From (4.11) it follows that

$$
\left\|f_{i k}\left(\cdot+y_{k}\right)\right\|_{W_{\infty}^{l-s_{i}, p}\left(\Omega_{k}\right)} \longrightarrow 0 \quad \text { as } k \longrightarrow \infty
$$

Hence the right-hand side in (4.24) tends to zero. Passing to the limit in this equation, we obtain

$$
\sum_{h=1}^{N} \sum_{|\alpha| \leq \alpha_{i h}} \hat{a}_{i h}^{\alpha}(y) D^{\alpha} w_{h 0}(y)=0, \quad y \in \Omega_{*}
$$

Consider now (4.17). From (4.20) it follows that $D^{\beta} w_{h k}\left(|\beta| \leq \beta_{i h}\right)$ tends to $D^{\beta} w_{h 0}$ in $W^{l-\sigma_{j}-\epsilon, p}\left(R^{n}\right)$ locally. Hence (3.17) implies that

$$
\sum_{h=1}^{N} \sum_{|\beta| \leq \beta_{j h}} b_{j h k}^{\beta}(y) D^{\beta} w_{h k}(y) \longrightarrow \sum_{h=1}^{N} \sum_{|\beta| \leq \beta_{j h}} \hat{b}_{j h}^{\beta}(y) D^{\beta} w_{h 0}(y)
$$

in $W_{\text {loc }}^{l-\sigma_{j}-\epsilon, p}\left(R^{n}\right)$. Therefore this convergence takes place also in $W_{\text {loc }}^{l-\sigma_{j}-\epsilon, p}\left(\Omega_{*}\right)$ and, consequently, in $W_{\mathrm{loc}}^{l-\sigma_{j}-\epsilon-1 / p, p}\left(\partial \Omega_{*}\right)$. In other words, we have proved that the convergence (4.28) is in $W_{\text {loc }}^{l-\sigma_{j}-\epsilon-1 / p, p}\left(\partial \Omega_{k} \rightarrow \partial \Omega_{*}\right)$ (see Definition 3.7). 
Consider next the right-hand side in (4.17). According to (4.11) we have

$$
\left\|f_{j k}^{b}\left(\cdot+y_{k}\right)\right\|_{W_{\infty}^{l-\sigma_{j}-1 / p, p}\left(\partial \Omega_{k}\right)} \longrightarrow 0 \quad \text { as } k \rightarrow \infty
$$

We can extend $f_{j k}^{b}\left(y+y_{k}\right)$ to the whole $R^{n}$ in such a way that

$$
f_{j k}^{b}\left(\cdot+y_{k}\right) \longrightarrow 0 \quad \text { in } W_{\infty}^{l-\sigma_{j}, p}\left(R^{n}\right)
$$

Therefore

$$
f_{j k}^{b}\left(\cdot+y_{k}\right) \longrightarrow 0 \quad \text { in } W_{\mathrm{loc}}^{l-\sigma_{j}-1 / p, p}\left(\partial \Omega_{k} \longrightarrow \partial \Omega_{*}\right)
$$

From this and convergence (4.28) it follows

$$
\sum_{h=1}^{N} \sum_{|\beta| \leq \beta_{j h}} \hat{b}_{j h}^{\beta}(y) D^{\beta} w_{h 0}(y)=0, \quad y \in \partial \Omega_{*} .
$$

From (4.22) it follows that the left-hand side of this equality belongs to $W_{\infty}^{l-\sigma_{j}-1 / p, p}\left(\partial \Omega_{*}\right)$. Hence it can be regarded as an equality in $W_{\infty}^{l-\sigma_{j}-1 / p, p}\left(\partial \Omega_{*}\right)$.

From (4.27) and (4.32) we conclude that $w_{0}$ is a solution of the limiting problem (4.23). We prove now that $w_{0} \neq 0$. From (4.8) and (4.12) we have

$$
\left\|w_{k}\right\|_{L^{p}\left(\Omega_{k} \cap Q_{0}\right)}>\frac{1}{2 c}
$$

where $Q_{0}$ is the unit ball with the center at the origin. We prove that

$$
\left\|w_{0}\right\|_{L^{p}\left(\Omega_{*} \cap Q_{0}\right)} \geq \frac{1}{2 c}
$$

Indeed, from (4.20),

$$
w_{k} \longrightarrow w_{0} \quad \text { in } L_{\mathrm{loc}}^{p}\left(R^{n}\right)
$$

Denote $S_{k}=\Omega_{k} \cap Q_{0}, S_{*}=\Omega_{*} \cap Q_{0}$. Then

$$
\begin{aligned}
& \left|\left\|w_{k}\right\|_{L^{p}\left(S_{k}\right)}-\left\|w_{0}\right\|_{L^{p}\left(S_{*}\right)}\right| \\
& \quad \leq\left|\left\|\left.w_{k}\right|_{L^{p}\left(S_{k}\right)}-\right\| w_{0}\right|_{L^{p}\left(S_{k}\right)}|+||| w_{0}\left\|_{L^{p}\left(S_{k}\right)}-\right\| w_{0} \|_{L^{p}\left(S_{*}\right)} \mid \\
& \quad \equiv A_{k}+B_{k} .
\end{aligned}
$$


748 Normal solvability of general linear elliptic problems

Further

$$
\begin{aligned}
A_{k} & \leq\left\|w_{k}-w_{0}\right\|_{L^{p}\left(S_{k}\right)}=\sum_{i=1}^{N}\left\|w_{i k}-w_{i 0}\right\|_{L^{p}\left(S_{k}\right)} \\
& =\sum_{i=1}^{N}\left(\int_{S_{k}}\left|w_{i k}-w_{i 0}\right|^{p} d x\right)^{1 / p} \longrightarrow 0 \quad \text { as } k \longrightarrow \infty, \\
B_{k} & \leq \sum_{i=1}^{N}\left|\left\|\left.w_{i 0}\right|_{L^{p}\left(S_{k}\right)}-|| w_{i 0}\right\|_{L^{p}\left(S_{*}\right)}\right| \\
& \leq M \sum_{i=1}^{N}\left(\int_{S_{k} \triangle S_{*}}\left|w_{i 0}\right|^{p} d x\right)^{1 / p} \longrightarrow 0 \quad \text { as } k \longrightarrow \infty
\end{aligned}
$$

since the measure of the symmetric difference $S_{k} \triangle S_{*}$ converges to 0 .

We have proved that

$$
\left\|w_{k}\right\|_{L^{p}\left(\Omega_{k} \cap Q_{0}\right)} \longrightarrow\left\|w_{0}\right\|_{L^{p}\left(\Omega_{*} \cap Q_{0}\right)}
$$

and (4.34) follows from (4.33).

Thus there exists a limiting problem with a nonzero solution. This contradicts Condition NS. The theorem is proved.

Denote

$$
\omega_{\mu}=e^{\mu \sqrt{1+|x|^{2}}}
$$

where $\mu$ is a real number.

Theorem 4.2. Let Condition NS be satisfied. Then there exist numbers $M_{0}>0, R_{0}>0$ and $\mu_{0}>0$ such that for all $\mu, 0<\mu<\mu_{0}$ the following estimate holds:

$$
\left\|\omega_{\mu} u\right\|_{E_{\infty}} \leq M_{0}\left(\left\|\omega_{\mu} L u\right\|_{F_{\infty}}+\left\|\omega_{\mu} u\right\|_{L^{p}\left(\Omega_{R_{0}}\right)}\right), \quad \forall u \in E_{\infty} .
$$

More complete proof of this theorem is given in [24].

Proof. According to (4.1) we have

$$
\left\|\omega_{\mu} u\right\|_{E_{\infty}} \leq M\left(\left\|L\left(\omega_{\mu} u\right)\right\|_{F_{\infty}}+\left\|\omega_{\mu} u\right\|_{L^{p}\left(\Omega_{R_{0}}\right)}\right) .
$$

By (1.8), $L=\left(A_{1}, \ldots, A_{N}, B_{1}, \ldots, B_{m}\right)$. Consider first the operator

$$
A_{i}\left(\omega_{\mu} u\right)=\sum_{k=1}^{N} \sum_{|\alpha| \leq \alpha_{i k}} a_{i k}^{\alpha}(x) D^{\alpha}\left(\omega_{\mu} u_{k}\right), \quad i=1, \ldots, N .
$$

We have

$$
A_{i}\left(\omega_{\mu} u\right)=\omega_{\mu} A_{i}(u)+\Phi_{i}
$$


where

$$
\Phi_{i}=\sum_{k=1}^{N} \sum_{|\alpha| \leq \alpha_{i k}} \sum_{\beta+\gamma=\alpha,|\beta|>0} a_{i k}^{\alpha}(x) c_{\beta \gamma} D^{\beta} \omega_{\mu} D^{\gamma} u_{k}
$$

and $c_{\beta \gamma}$ are some constants. Direct calculations give the following estimate

$$
\left\|\Phi_{i}\right\|_{W_{\infty}^{l-s_{i}, p}} \leq M_{1} \mu\left\|\omega_{\mu} u\right\|_{E_{\infty}(\Omega)} .
$$

For the boundary operators we have

$$
B_{j}\left(\omega_{\mu} u\right)=\sum_{k=1}^{N} \sum_{|\beta| \leq \beta_{j k}} b_{j k}^{\beta}(x) D^{\beta}\left(\omega_{\mu} u_{k}\right) .
$$

As above we get

$$
\begin{gathered}
B_{j}\left(\omega_{\mu} u\right)=\omega_{\mu} B_{j}(u)+\Psi_{j}, \\
\left\|\Psi_{j}\right\|_{W_{\infty}^{l-\sigma_{j}-1 / p, p}} \leq M_{2} \mu\left\|\omega_{\mu} u\right\|_{E_{\infty}(\Omega)} .
\end{gathered}
$$

From (4.43), (4.45), and (4.47) we obtain

$$
\left\|L\left(\omega_{\mu} u\right)\right\|_{F_{\infty}} \leq\left\|\omega_{\mu} L u\right\|_{F_{\infty}}+M \mu\left\|\omega_{\mu} u\right\|_{E_{\infty}} .
$$

The assertion of the theorem follows from this estimate and (4.41). The theorem is proved.

Corollary 4.3. If $0<\mu<\mu_{0}, u \in E_{\infty}$, and $\omega_{\mu} L u \in F_{\infty}$, then $\omega_{\mu} u \in E_{\infty}$. In particular, if $u \in E_{\infty}$ and $L u=0$, then $\omega_{\mu} u \in E_{\infty}$.

\section{Normal solvability}

We recall that an operator $L$ acting in Banach spaces is normally solvable if its range is closed. It is called $n$-normally solvable if it is normally solvable and has a finite dimensional kernel (see, e.g., [7])

Theorem 5.1. Let Condition NS be satisfied. Then the elliptic operator $L: E_{\infty}(\Omega) \rightarrow F_{\infty}(\Omega)$ is normally solvable and has a finite dimensional kernel.

Proof. It is known that a linear operator has a finite dimensional kernel and a closed range if and only if its restriction to any bounded closed set is proper.

Let $L u_{n}=f_{n}, u_{n} \in E_{\infty}(\Omega), f_{n} \in F_{\infty}(\Omega)$. Suppose that $\left\|u_{n}\right\|_{E_{\infty}} \leq M$ and $f_{n}$ is convergent. It is sufficient to prove that the sequence $u_{n}$ is compact. This follows from Theorem 4.1. The theorem is proved.

In the next theorem we prove that Condition NS is necessary for the operator $L$ to be normally solvable with a finite dimensional kernel. To simplify the construction we impose a stronger regularity condition on the boundary of the domain, $\partial \Omega \in C^{r+1+\theta}$. We will use the following lemma. 
Lemma 5.2. Let $\Omega_{k}$ and $\Omega_{*}$ be a shifted and a limiting domains, respectively. Then for any $N$ there exists $k_{0}$ such that for $k>k_{0}$ there exists a diffeomorphism

$$
h_{k}(x): \bar{\Omega}_{k} \bigcap B_{N} \longrightarrow \bar{\Omega}_{*} \bigcap B_{N}
$$

satisfying the condition

$$
\left\|h_{k}(x)-x\right\|_{C^{l+\theta_{0}}\left(\bar{\Omega}_{k} \cap B_{N}\right)} \longrightarrow 0
$$

as $k \rightarrow \infty$. Here $0<\theta_{0}<\theta$.

The proof is given in [22]

Theorem 5.3. Suppose that a limiting problem for the operator $L$ has a nonzero solution. Then the operator $L$ is not n-normally solvable.

Explanation. To prove the theorem we construct a sequence $u_{n}$ such that it is not compact in $E_{\infty}(\Omega)$ but $L u_{n}$ converges to zero in $F_{\infty}(\Omega)$. The idea of the construction is rather simple but its technical realization is rather long. This is why we preface the proof by a short description of the construction.

Let us consider a ball $B_{R}\left(x_{k}\right)$ of a fixed radius $R$ with the center at $x_{k}$. From the definition of limiting problems it follows that we can choose the sequence $x_{k}$ in such a way that inside $B_{R}\left(x_{k}\right)$ the domain $\Omega$ is close to the limiting domain, and the coefficients of the operator are close to the coefficients of the limiting operator. Moreover, the domain and the coefficients converge to their limits as $k \rightarrow \infty$. Thus we move the ball $B_{R}\left(x_{k}\right)$ to infinity and superpose it on the domain $\Omega$ in the places where the operator and the domain are close to their limits and converge to them.

If $u_{0}$ is a nonzero solution of the limiting problem, then we shift it to the ball $B_{R}\left(x_{k}\right)$. Denote the shifted function by $u_{k}$. Then inside $B_{R}\left(x_{k}\right), L u_{k}$ tends to zero as $k \rightarrow \infty$. The sequence $u_{k}$ is not compact.

If $u_{0}$ had a bounded support, the construction would be finished. Since it is not necessarily the case, we multiply $u_{0}$ by an infinitely differentiable function $\phi$ with a bounded support. Of course, this product is not an exact solution of the limiting problem any more. However, all terms of the difference $\hat{L}\left(\phi u_{0}\right)-\phi \hat{L} u_{0}$ contain derivatives of $\phi$. If the support of $\phi$ is sufficiently large, then the derivatives of $\phi$ can be done sufficiently small. Hence when we move the ball $B_{R}\left(x_{k}\right)$ to infinity, we should also increase its radius and also increase supports of functions $\phi_{k}$.

Proof. Suppose that there exists a limiting operator $\hat{L}$ such that

$$
\hat{L} u_{0}=0, \quad u_{0} \in E_{\infty}(\Omega), u_{0} \neq 0 .
$$

Consider an infinitely differentiable function $\varphi(x), x \in R^{n}$ such that $0 \leq \varphi(x) \leq 1$, $\varphi(x)=1$ for $|x|<1, \varphi(x)=0$ for $|x|>2$. If $\left\{x_{k}\right\}$ is the sequence for which the limiting operator $\hat{L}$ is defined, denote

$$
\varphi_{k}(x)=\varphi\left(\frac{x}{r_{k}}\right),
$$


where $r_{k} \rightarrow \infty$ and $r_{k} \leq\left|x_{k}\right| / 3$. Some other conditions on the sequence $r_{k}$ will be formulated below.

Let $V_{j}=\left\{y: y \in R^{n},|y|<j\right\}, j=1,2, \ldots$ Denote by $n_{j}$ a number such that for $k \geq n_{j}$ the diffeomorphism $h_{k}$ defined in Lemma 5.2 can be constructed in $\Omega_{k} \cap V_{j+1}$ and

$$
\left\|h_{k}(y)-y\right\|_{C^{l+\theta_{0}}\left(\Omega_{k} \cap V_{j+1}\right)}<\delta,
$$

where $\delta>0$ is taken so small that $\left|h_{k}^{\prime}-I\right|<1 / 2, h_{k}^{\prime}$ is the Jacobian matrix and $I$ is the identity matrix.

For arbitrary $k_{j} \geq n_{j}$ we take $r_{k_{j}}=\min \left(j / 2,\left|x_{k_{j}}\right| / 3\right)$. Let

$$
\begin{gathered}
v_{k_{j}}(y)=\varphi_{k_{j}}(y) u_{0}\left(h_{k_{j}}(y)\right) \quad \text { for } y \in \Omega_{k_{j}} \bigcap V_{j+1}, \\
v_{k_{j}}(y)=0 \quad \text { for } y \in \Omega_{k_{j}},|y| \geq j+1
\end{gathered}
$$

Denote

$$
u_{k_{j}}(x)=v_{k_{j}}\left(x-x_{k_{j}}\right), \quad x \in \Omega .
$$

It is easy to see that $u_{k_{j}} \in E_{\infty}(\Omega)$ and

$$
\left\|u_{k_{j}}\right\|_{E_{\infty}(\Omega)} \leq M,
$$

where $M$ does not depend on $k_{j}$. Indeed, obviously

$$
\varphi_{k_{j}}(y)=0
$$

for $y$ outside $V_{j}$. Therefore to prove (5.8) it is sufficient to show that

$$
\left\|v_{k_{j}}\right\|_{E_{\infty}\left(\Omega_{k_{j}} \cap v_{j+1}\right)} \leq M_{1}
$$

or

$$
\left\|u_{0}\left(h_{k_{j}}(y)\right)\right\|_{E_{\infty}\left(\Omega_{k_{j}} \cap V_{j+1}\right)} \leq M_{2}
$$

where $M_{1}$ and $M_{2}$ do not depend on $k_{j}$. This follows from (5.5) and the fact that $u_{0} \in$ $E_{\infty}\left(\Omega_{*}\right)$.

We prove now that the choice of $k_{j}$ in (5.7) can be specified in such a way that

(i) $L u_{k_{j}} \rightarrow 0$ in $F_{\infty}(\Omega)$ as $k_{j} \rightarrow \infty$,

(ii) the sequence $\left\{u_{k_{j}}\right\}$ is not compact in $E_{\infty}(\Omega)$.

The assertion of the theorem will follow from this.

(i) We consider first the operators $A_{i}, i=1, \ldots, N$, and then the operator $B_{j}, j=1, \ldots, m$. For any $k=k_{j} \geq n_{j}$ we have

$$
A_{i} u_{k}=A_{i}^{1} u_{k}+A_{i}^{2} u_{k},
$$

where

$$
A_{i}^{1} u_{k}(x)=\varphi_{k}\left(x-x_{k}\right) \sum_{r=1}^{N} \sum_{|\alpha| \leq \alpha_{i r}} a_{i r}^{\alpha}(x) D^{\alpha} u_{0 r}\left(h_{k}\left(x-x_{k}\right)\right), \quad x \in \Omega
$$


752 Normal solvability of general linear elliptic problems

and $A_{i}^{2}$ contains derivatives of $\varphi_{k}$. Obviously

$$
\left\|A_{i}^{2} u_{k}\right\|_{W_{\infty}^{l-s_{i}, p}(\Omega)} \longrightarrow 0
$$

as $k \rightarrow \infty$.

Denote $y=x-x_{k}$. From (5.13) we obtain

$$
A_{i}^{1} u_{k}\left(y+x_{k}\right)=\varphi_{k}(y) T_{i k}(y), \quad y \in \Omega_{k},
$$

where

$$
T_{i k}(y)=\sum_{r=1}^{N} \sum_{|\alpha| \leq \alpha_{i r}} a_{i r k}^{\alpha}(y) D^{\alpha} u_{0 r}\left(h_{k}(y)\right), \quad y \in \Omega_{k},
$$

$a_{i r k}^{\alpha}(y)=a_{i r}^{\alpha}\left(y+x_{k}\right)$. We prove that for any fixed $j$

$$
\left\|T_{i k}\right\|_{W_{\infty}^{l-s_{i}, p}\left(\Omega_{k} \cap V_{j+1}\right)} \longrightarrow 0
$$

as $k \rightarrow \infty$. Indeed, by the definition of $u_{0}$ the following equality holds:

$$
\sum_{r=1}^{N} \sum_{|\alpha| \leq \alpha_{i r}} \hat{a}_{i r}^{\alpha}(x) D^{\alpha} u_{0 r}(x)=0, \quad x \in \Omega_{*} .
$$

Here $\hat{a}_{i r}^{\alpha}(x)$ are the limiting coefficients. Hence

$$
T_{i k}(y)=\sum_{r=1}^{N} \sum_{|\alpha| \leq \alpha_{i r}}\left[S_{i r k}^{\alpha}(y)+P_{i r k}^{\alpha}(y)\right]
$$

where

$$
\begin{gathered}
S_{i r k}^{\alpha}(y)=a_{i r k}^{\alpha}(y)\left[D_{y}^{\alpha} u_{0 r}\left(h_{k}(y)\right)-D_{x}^{\alpha} u_{0 r}\left(h_{k}(y)\right)\right], \\
P_{i r k}^{\alpha}(y)=\left[a_{i r k}^{\alpha}(y)-\hat{a}_{i r}^{\alpha}\left(h_{k}(y)\right)\right] D_{x}^{\alpha} u_{0 r}\left(h_{k}(y)\right) .
\end{gathered}
$$

The first factor in the right-hand side of (5.20) is bounded in the norm $C^{l-s_{i}}\left(\Omega_{k}\right)$ since

$$
\left\|a_{i r k}^{\alpha}\right\|_{C^{l-s_{i}}\left(\Omega_{k}\right)}=\left\|a_{i r}^{\alpha}\right\|_{C^{l-s_{i}}(\Omega)} .
$$

From Lemma 5.2 it follows that the second factor tends to 0 in the norm $W_{\infty}^{l-s_{i}, p}\left(\Omega_{k} \cap V_{j+1}\right)$ as $k \rightarrow \infty$. Consequently,

$$
\left\|S_{i r k}^{\alpha}\right\|_{W_{\infty}^{l-s_{i}, p}\left(\Omega_{k} \cap V_{j+1}\right)} \longrightarrow 0 \quad \text { as } k \longrightarrow \infty
$$


Consider (5.21). Using (5.5) we easily prove that

$$
\left\|D_{x}^{\alpha} u_{0}\left(h_{k}(y)\right)\right\|_{W_{\infty}^{l-s_{i}, p}\left(\Omega_{k} \cap V_{j+1}\right)} \leq M_{3}
$$

with $M_{3}$ independent of $k$.

To prove (5.17) it remains to show that

$$
\left\|a_{i r k}^{\alpha}(\cdot)-\hat{a}_{i r}^{\alpha}\left(h_{k}(\cdot)\right)\right\|_{C^{l-s_{i}}\left(\Omega_{k} \cap V_{j+1}\right)} \longrightarrow 0 \quad \text { as } k \rightarrow \infty .
$$

We recall that it is supposed that $a_{i r k}^{\alpha}(y)$ and $\hat{a}_{i r}^{\alpha}(y)$ are defined for $y \in R^{n}$,

$$
\left\|a_{i r k}^{\alpha}\right\|_{C^{l-s_{i}+\theta}\left(R^{n}\right)} \leq M
$$

with $M$ independent of $k, \hat{a}_{i r}^{\alpha}(y) \in C^{l-s_{i}+\theta}\left(R^{n}\right)$ and

$$
a_{i r k}^{\alpha}(y) \longrightarrow \hat{a}_{i r}^{\alpha}(y)
$$

in $C_{\mathrm{loc}}^{l-s_{i}}\left(R^{n}\right)$ as $k \rightarrow \infty$. We have

$$
\begin{aligned}
& \left\|a_{i r k}^{\alpha}(y)-\hat{a}_{i r}^{\alpha}\left(h_{k}(y)\right)\right\|_{C^{l-s_{i}}\left(\Omega_{k} \cap V_{j+1}\right)} \\
& \quad \leq\left\|a_{i r k}^{\alpha}(y)-\hat{a}_{i r}^{\alpha}(y)\right\|_{C^{l-s_{i}}\left(\Omega_{k} \cap V_{j+1}\right)}+\left\|\hat{a}_{i r}^{\alpha}(y)-\hat{a}_{i r}^{\alpha}\left(h_{k}(y)\right)\right\|_{C^{l-s_{i}}\left(\Omega_{k} \cap V_{j+1}\right)} .
\end{aligned}
$$

The first term on the right tends to zero as $k \rightarrow \infty$ according to (5.27). The second term tends to zero by the properties of the function $\hat{a}_{i r}^{\alpha}$ mentioned above, by Lemma 5.2 and by inequality (5.5). Thus (5.17) is proved.

Now we specify the choice of $k_{j}$ in (5.7). According to (5.17) for any $j$ we can take $p_{j}$ in such a way that

$$
\left\|T_{i k}\right\|_{W_{\infty}^{l-s_{i}, p}\left(\Omega_{k} \cap V_{j+1}\right)}<\frac{1}{j}
$$

for $k \geq p_{j}$. We put $k_{j}=\max \left(n_{j}, p_{j}\right)$. Then obviously

$$
\left\|\varphi_{k_{j}} T_{i k_{j}}\right\|_{W_{\infty}^{l-s_{i}, p}\left(\Omega_{k_{j}}\right)} \longrightarrow 0 \quad \text { as } k \longrightarrow \infty
$$

Consider now the boundary operators $B_{i}$. According to our assumptions, the coefficients $b_{i h}^{\beta}(x)$ of the operators $B_{i}(i=1, \ldots, m)$ are defined in the domain $\bar{\Omega}$ and belong to the space $C^{l-\sigma_{i}+\theta}(\bar{\Omega})$. By the same arguments, which we used for the operator $A_{i}$, we prove that

$$
\left\|B_{i} u_{k_{j}}\right\|_{W_{\infty}^{l-\sigma_{i}-1 / p, p}(\partial \Omega)} \longrightarrow 0 \quad \text { as } k_{j} \longrightarrow \infty
$$

We repeat the same construction as above and obtain the following operator:

$$
T_{i k}(y)=\sum_{h=1}^{N} \sum_{|\beta| \leq \beta_{i h}} b_{i h_{k}}^{\beta}(y) D^{\beta} u_{0 h}\left(h_{k}(y)\right), \quad y \in \Omega_{k},
$$


754 Normal solvability of general linear elliptic problems

where $b_{i h k}^{\beta}(y)=b_{i h}^{\beta}\left(y+x_{k}\right)$. We prove that

$$
\left\|T_{i k}\right\|_{W_{\infty}^{l-\sigma_{i}-1 / p, p}\left(\partial \Omega_{k} \cap V_{j+1}\right)} \longrightarrow 0
$$

Indeed, denote

$$
g_{i}(x)=\sum_{h=1}^{N} \sum_{|\beta| \leq \beta_{\text {ih }}} \hat{b}_{i h} \beta(y) D^{\beta} u_{0 h}(x), \quad x \in \Omega_{*} .
$$

This expression equals 0 only at the boundary $\partial \Omega_{*}$. Therefore instead of what is written above for the operator $A_{i}$, we have now

$$
T_{i k}(y)=Q_{i k}(y)+g_{i}\left(h_{k}(y)\right)
$$

where

$$
Q_{i k}(y)=\sum_{h=1}^{N} \sum_{|\beta| \leq \beta_{i h}}\left[S_{i h k}^{\beta}(y)+P_{i h k}^{\beta}(y)\right] .
$$

Here $S$ and $P$ are the same as for the operator $A$ but the coefficients $a$ are replaced by $b$. Exactly as we have done for the operator $A$ we prove that

$$
\left\|Q_{k}\right\|_{W_{\infty}^{l-\sigma_{i}, p}\left(\Omega_{k} \cap V_{j+1}\right)} \longrightarrow 0 \quad \text { as } k \longrightarrow \infty
$$

It follows that

$$
\left\|Q_{k}\right\|_{W_{\infty}^{l-\sigma_{i}-1 / p, p}\left(\partial \Omega_{k} \cap V_{j+1}\right)} \longrightarrow 0 \quad \text { as } k \longrightarrow \infty .
$$

Since for $y \in \partial \Omega_{k}$ we have $h_{k}(y) \in \partial \Omega_{*}$, we have $g_{i}\left(h_{k}(y)\right)=0$ for $y \in \partial \Omega_{k}$. From this, (5.35) and (5.38) we get (5.33). Thus the assertion (i) is proved.

(ii) We prove now that sequence (5.7) does not have a convergent subsequence. Obviously $u_{k_{j}}(x)=0$ for $|x|<r_{k_{j}}$ and, consequently,

$$
\int_{\Omega} u_{k_{j}}(x) \omega(x) d x \longrightarrow 0
$$

as $k_{j} \rightarrow \infty$ for any continuous $\omega(x)$ with a compact support.

For any subsequence $s_{i}$ of $k_{j}$ there exists $N$ such that

$$
\int_{\Omega}\left|u_{s_{i}}(x)\right|^{p} d x \geq \rho
$$


for $s_{i}>N$ and some $\rho>0$. Indeed, let $y=x-x_{s_{i}}$. Then

$$
\begin{aligned}
T_{i} & \equiv \int_{\Omega}\left|u_{s_{i}}(x)\right|^{p} d x=\int_{\Omega_{s_{i}}}\left|v_{s_{i}}(y)\right|^{p} d y \\
& =\int_{\Omega_{s_{i}} \cap V_{j+1}}\left|\varphi_{s_{i}}(y) u_{0}\left(h_{s_{i}}(y)\right)\right|^{p} d y \\
& \geq \int_{\Omega_{s_{i}} \cap V_{r_{s_{i}}}}\left|u_{0}\left(h_{s_{i}}(y)\right)\right|^{p} d y .
\end{aligned}
$$

We do the change of variables $y=h_{s_{i}}^{-1}(x)$ in the last integral. Then

$$
T_{i} \geq \int_{\Omega_{*} \cap W_{s_{i}}}\left|u_{0}(x)\right|^{p}\left|\frac{d h_{s_{i}}^{-1}(x)}{d x}\right| d x,
$$

where $W_{s_{i}}=h_{s_{i}}\left(V_{r_{s_{i}}}\right)$.

Since $\left\|u_{0}\right\|_{L^{p}\left(\Omega_{*}\right)} \neq 0$, there exists a ball $B_{l}=\{x:|x|<l\}$ and a number $\rho_{0}>0$ such that

$$
\int_{\Omega_{*} \cap B_{l}}\left|u_{0}(x)\right|^{p} d x \geq \rho_{0} .
$$

Increasing $N$, if necessary, we can suppose that $B_{l} \subset W_{s_{i}}$ and $\left|d h_{s_{i}}^{-1}(x) / d x\right| \geq \varepsilon$ for $x \in B_{l}$ and some $\varepsilon>0$. The last inequality follows from the fact that according to (5.5) the derivatives of $h_{s_{i}}(y)$ are uniformly bounded. By (5.43) we get $T_{i} \geq \varepsilon \rho_{0}$ and (5.40) is proved.

If (5.7) has a convergent subsequence: $u_{s_{i}} \rightarrow u_{*}$ in $E(\Omega)$, then this convergence is also in $L^{p}(\Omega)$. From (5.39) it follows that $u_{*}=0$ which contradicts (5.40). Thus the sequence (5.7) is not compact in $E(\Omega)$. The theorem is proved.

\section{References}

[1] S. Agmon, A. Douglis, and L. Nirenberg, Estimates near the boundary for solutions of elliptic partial differential equations satisfying general boundary conditions. II, Comm. Pure Appl. Math. 17 (1964), 35-92.

[2] M. S. Agranovich, Elliptic boundary problems, Partial Differential Equations, IX, Encyclopaedia Math. Sci., vol. 79, Springer, Berlin, 1997, pp. 1-144, 275-281.

[3] M. S. Agranovich and M. I. Višik, Elliptic problems with a parameter and parabolic problems of general type, Uspehi Mat. Nauk 19 (1964), no. 3 (117), 53-161 (Russian), English translation in Russian Math. Surveys 19 (1964), no. 3, 53-157.

[4] C. Barillon and V. A. Volpert, Topological degree for a class of elliptic operators in $\mathbf{R}^{n}$, Topol. Methods Nonlinear Anal. 14 (1999), no. 2, 275-293.

[5] A. Douglis and L. Nirenberg, Interior estimates for elliptic systems of partial differential equations, Comm. Pure Appl. Math. 8 (1955), 503-538.

[6] J. Favard, Sur les équations différentielles linéaires à coefficients presque-périodiques, Acta Math. 51 (1928), 31-81 (French).

[7] S. G. Krein, Linear Differential Equations in Banach Space, Translations of Mathematical Monographs, vol. 29, American Mathematical Society, Rhode Island, 1971, translated from the Russian by J. M. Danskin.

[8] B. M. Levitan, Almost Periodic Functions, Gosudarstv. Izdat. Tehn.-Teor. Lit., Moscow, 1953.

[9] B. M. Levitan and V. V. Zhikov, Almost Periodic Functions and Differential Equations, Cambridge University Press, New York, 1982, translated from the Russian by L. W. Longdon. 
[10] J.-L. Lions and E. Magenes, Problèmes aux limites non homogènes et applications. Vol. 1, Travaux et Recherches Mathématiques, no. 17, Dunod, Paris, 1968.

[11] R. B. Lockhart, Fredholm properties of a class of elliptic operators on noncompact manifolds, Duke Math. J. 48 (1981), no. 1, 289-312.

[12] È. M. Mukhamadiev, Normal solvability and noethericity of elliptic operators in spaces of functions on $\mathbf{R}^{n}$. I, Zap. Nauchn. Sem. Leningrad. Otdel. Mat. Inst. Steklov. (LOMI) 110 (1981), 120140, 244 (Russian), English translation in J. Soviet Math. 25 (1984), no. 1, 884-901.

[13] - Normal solvability and noethericity of elliptic operators in spaces of functions on $\mathbf{R}^{n}$. II, Zap. Nauchn. Sem. Leningrad. Otdel. Mat. Inst. Steklov. (LOMI) 138 (1984), 108-126 (Russian), English translation in J. Soviet Math. 32 (1986), no. 5, 499-512.

[14] A. A. Pankov, Bounded and almost Periodic Solutions of Nonlinear Operator Differential Equations, Mathematics and Its Applications (Soviet Series), vol. 55, Kluwer Academic Publishers, Dordrecht, 1990.

[15] V. S. Rabinovich, The Fredholm property of general boundary value problems on noncompact manifolds, and limit operators, Dokl. Akad. Nauk 325 (1992), no. 2, 237-241 (Russian), English translation in Russian Acad. Sci. Dokl. Math. 46 (1993), no. 1, 53-58.

[16] E. Schrohe, Fréchet algebra techniques for boundary value problems on noncompact manifolds: Fredholm criteria and functional calculus via spectral invariance, Math. Nachr. 199 (1999), $145-185$.

[17] M. A. Šubin, The Favard-Muhamadiev theory, and pseudodifferential operators, Dokl. Akad. Nauk SSSR 225 (1975), no. 6, 1278-1280 (Russian).

[18] Almost periodic functions and partial differential operators, Russian Math. Surveys 33 (1978), no. 2, 1-52.

[19] M. I. Višik and G. I. Èskin, Normally solvable problems for elliptic systems of convolution equations, Mat. Sb. (N.S.) 74 (116) (1967), 326-356 (Russian), English translation in Math. USSR Sb. 3 (1967), 303-330.

[20] L. R. Volevič, Solvability of boundary value problems for general elliptic systems, Mat. Sb. (N.S.) 68 (110) (1965), 373-416 (Russian), English translation in Amer. Math. Soc. Transl. Ser. 2 67 (1968), 182-225.

[21] V. A. Volpert, The spectrum of an elliptic operator in an unbounded cylindrical domain, Dokl. Akad. Nauk Ukrain. SSR Ser. A (1981), no. 9, 9-12, 94 (Russian).

[22] A. I. Volpert and V. A. Volpert, Normal solvability and properness of elliptic problems, Partial Differential Equations (M. Agranovich and M. Shubin, eds.), Amer. Math. Soc. Transl. Ser. 2, vol. 206, American Mathematical Society, Rhode Island, 2002, pp. 193-237.

[23] V. A. Volpert and A. I. Volpert, Properness and topological degree for general elliptic operators, Abstr. Appl. Anal. 2003 (2003), no. 3, 129-181.

[24] A. Volpert and V. Volpert, Fredholm property of elliptic operators in unbounded domains, to appear in Trans. Moscow Math. Soc.

[25] A. I. Volpert, V. A. Volpert, and V. A. Volpert, Traveling Wave Solutions of Parabolic Systems, Translations of Mathematical Monographs, vol. 140, American Mathematical Society, Rhode Island, 1994, translated from the Russian manuscript by James F. Heyda.

[26] H. F. Walker, A Fredholm theory for a class of first-order elliptic partial differential operators in $\mathbf{R}^{n}$, Trans. Amer. Math. Soc. 165 (1972), 75-86.

A. Volpert: Department of Mathematics, Technion-Israel Institute of Technology, 32000 Haifa, Israel

E-mail address:volp@tx.technion.ac.il

V. Volpert: Laboratoire de Mathématiques Appliquées, UMR 5585 CNRS, Université Lyon 1, 69622 Villeurbanne, France 


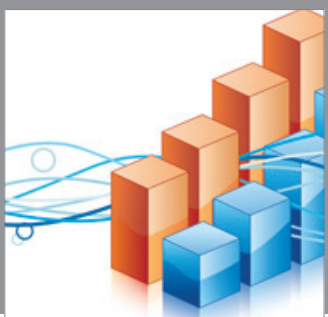

Advances in

Operations Research

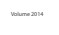

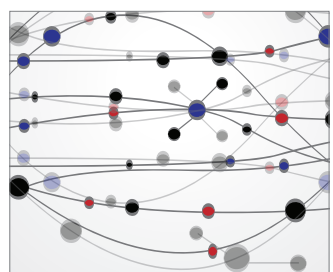

\section{The Scientific} World Journal
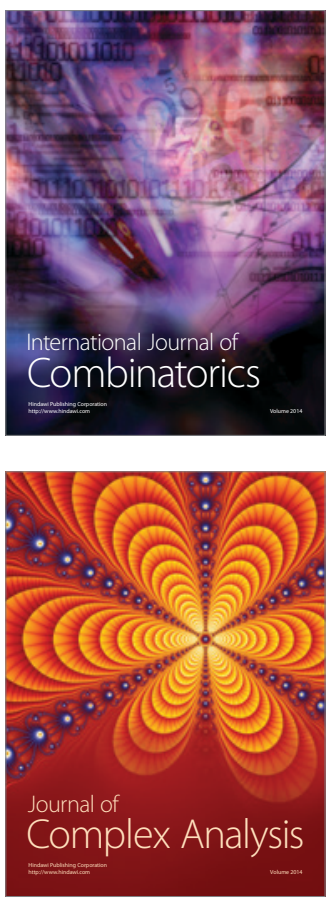

International Journal of

Mathematics and

Mathematical

Sciences
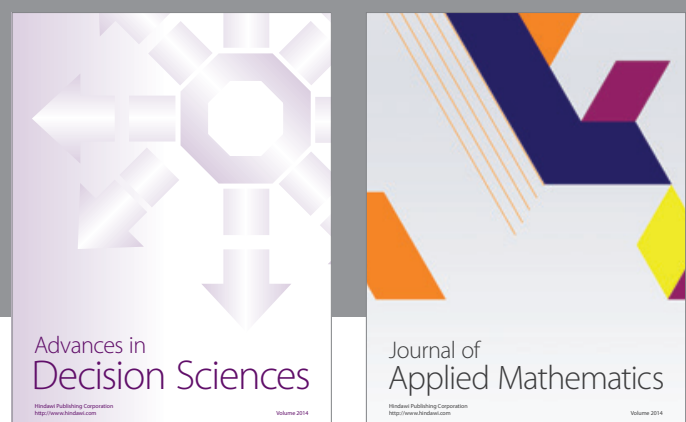

Journal of

Applied Mathematics
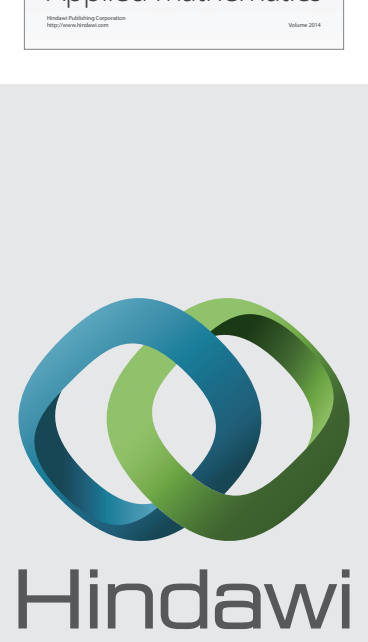

Submit your manuscripts at http://www.hindawi.com
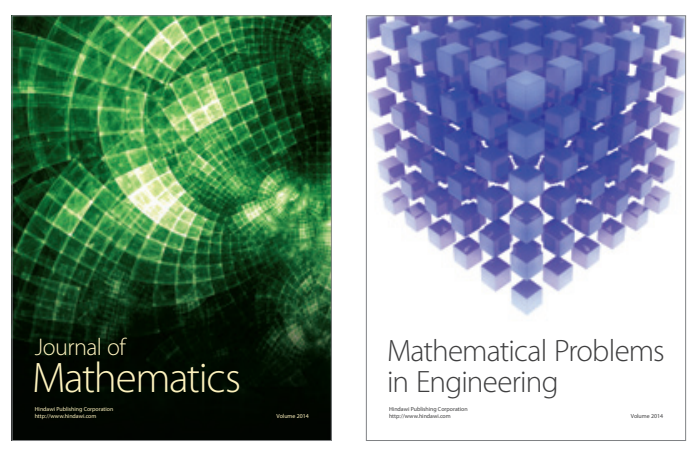

Mathematical Problems in Engineering
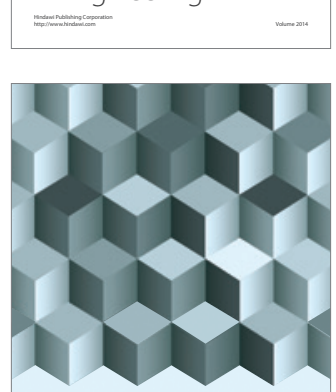

Journal of

Function Spaces
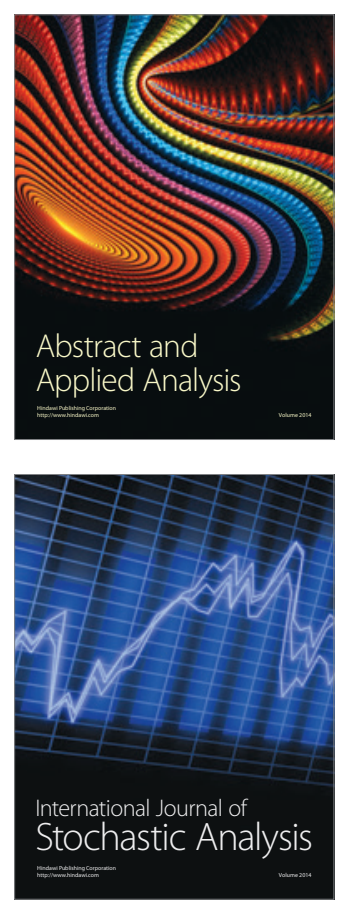

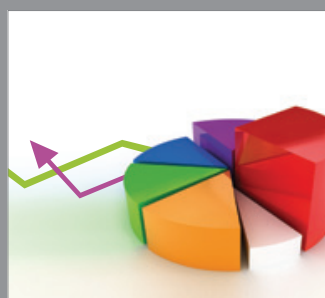

ournal of

Probability and Statistics

Promensencen
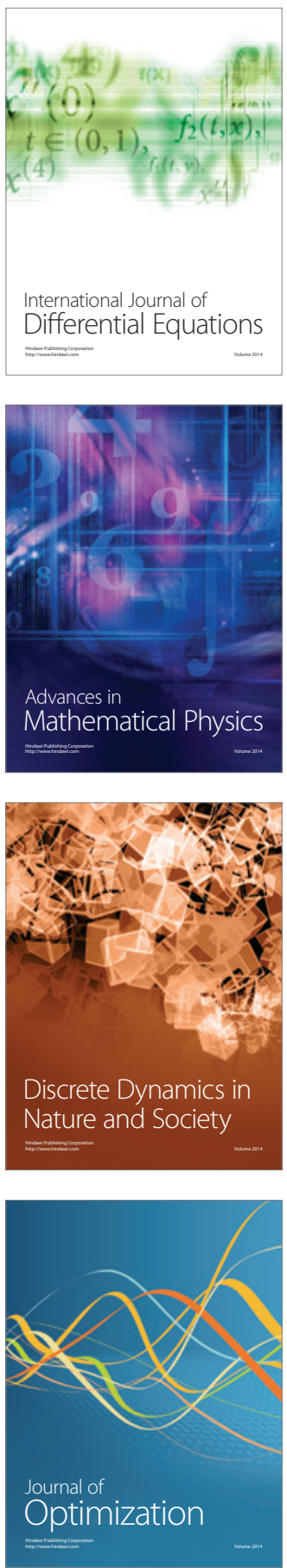